\title{
Clinical outcome of ultrasound-guided atelocollagen injection for patients with partial rotator cuff tear in an outpatient clinic: a preliminary study
}

\author{
Sang Hoon Chae, Jae Yeon Won, Jae Chul Yoo \\ Department of Orthopedic Surgery, Samsung Medical Center, Sungkyunkwan University School of Medicine, Seoul, Korea
}

\begin{abstract}
Background: Atelocollagen has been studied for restoration of rotator cuff tendon. In this study, we attempted to evaluate the clinical outcome of ultrasound-guided atelocollagen injection in an outpatient clinic for patients with partial rotator cuff tear.

Methods: We recruited 42 outpatients who visited our hospital from May 2019 to September 2019. Atelocollagen injection was performed in patients with partial rotator cuff tear diagnosed by magnetic resonance imaging and ultrasound. American Shoulder and Elbow Surgeons (ASES), Constant, Korean Shoulder Score (KSS) and Simple Shoulder Test (SST) scores, and range of motion were assessed before injection and after 2 months. Statistically, we analyzed the clinical results using the Wilcoxon signed-rank test.

Results: Finally, 15 patients were enrolled for analysis. There was no significant difference between pre- and post-injection in terms of range of motion, ASES (57.0 vs. 60.4), Constant (56.4 vs. 58.9), KSS (64.6 vs. 68.5), and pain-visual analog scale (4.2 vs. 3.7$)$, except function-visual analog scale (F-VAS; 6.3 vs. 7.1) and SST (6.6 vs. 6.9). A significant difference was found in SST ( $\mathrm{P}=0.046)$ and F-VAS (P=0.009). According to the ultrasound results at 2 months, we found hyperechoic materials in three of seven patients. The most common complication of atelocollagen injection was post-injection pain $(53 \%, 8 / 15)$.

Conclusions: Ultrasound-guided atelocollagen injection for partial rotator cuff tear showed no significant change in terms of clinical outcomes, except for F-vas and SST score. Tendon regeneration was not clear due to the remnants of atelocollagen present at 2-month follow-up ultrasound. There seems to be alarming post-injection pain for 2 to 3 days in the patients who received atelocollagen injection in an outpatient clinic.
\end{abstract}

Keywords: Atelocollagen; Partial rotator cuff tear; Ultrasound-guided injection; Preliminary study

\section{INTRODUCTION}

Partial thickness rotator cuff tear, which can be divided into bursal side tear, intra-tendinous tear, and articular side tear, has been reported to be more common and painful than full thickness tear with a prevalence rate ranging from $13 \%$ to $32 \%$ in the adult population [1-3]. Previous studies have shown that $80 \%$ of partial rotator cuff tears deteriorate or progress to full thickness rupture through conservative treatment [4]. Thus, for regeneration of rotator cuff tear, numerus strategies such as platelet-rich plasma and tissue engineering have been proposed.

Atelocollagen, which is obtained from bovine dermis, is central to one of the treatment strategies. Atelocollagen has favorable properties, including that it does not readily dissolve in the living

Received: February 8, 2020 Revised: March 17, 2020 Accepted: March 17, 2020

Correspondence to: Jae Chul Yoo

Department of Orthopedic Surgery, Samsung Medical Center, Sungkyunkwan University School of Medicine, 81 Irwon-ro, Gangnam-gu, Seoul 06351, Korea Tel: +82-2-3410-3501, Fax: +82-2-3410-0061, E-mail: shoulderyoo@gmail.com, ORCID: https://orcid.org/0000-0001-8378-1583

Financial support: None.

Conflict of interest: None.

Copyright@ 2020 Korean Shoulder and Elbow Society. All Rights Reserved.

This is an Open Access article distributed under the terms of the Creative Commons Attribution Non-Commercial License (http://creativecommons.org/licenses/by-nc/4.0/) which permits unrestricted non-commercial use, distribution, and reproduction in any medium, provided the original work is properly cited. 
body and offers low immunogenicity [5]. Therefore, it had been used as a scaffold of cellular proliferation with chondrocyte and mesenchymal stem cells embedded within its matrix [6,7]. In 2017, Suh et al. [8] demonstrated that patch-type atelocollagen can enhance the healing of rotator cuff tear in a rabbit model. However, to the best of our knowledge, there has been no study analyzing the clinical outcome of ultrasound-guided atelocollagen (gel-type) injection in an outpatient clinic. Therefore, in this preliminary study, we attempted to determine clinical outcomes of atelocollagen injection for patients with partial rotator cuff tear and complications observed during 2 months of follow-up.

\section{METHODS}

\section{Patient Selection}

This study was approved by the Institutional Review Board of Samsung Medical Center (IRB No. 2020-01-039-001), and informed consent was exempted because of the retrospective study design. We performed a chart review on prospectively collected data from 42 patients who underwent gel-type atelocollagen (Tendoregen; Phammode, Seoul, Korea) injection for partial rotator cuff tear. From May 2019 to September 2019, we performed ultrasound-guided atelocollagen injection in patients with partial rotator cuff tear. The inclusion criteria were (1) age 30 to 70 years, (2) articular-side, bursal-side, or intra-tendinous tear diagnosed by magnetic resonance imaging (MRI; $n=12$ ) or ultra- sound ( $\mathrm{n}=3$ ), and (3) agreed to nonsurgical treatment with atelocollagen. The exclusion criteria were (1) frozen shoulder, (2) follow-up loss at 2 months, (3) previous steroid injection within 3 months, (4) past medical history of allergic reactions or hypersensitivity on collagen, and (5) previous shoulder surgery. In addition, the initial 10 patients were excluded to avoid technical bias. We divided patients into three groups according to location of partial rotator cuff tear: (1) articular-side tear, (2) intra-tendinous tear, and (3) bursal-side tear (Fig. 1).

\section{Procedures}

Ultrasound-guided atelocollagen injection procedures were carried out by three orthopaedic surgeons who underwent fellowship training in the shoulder. The atelocollagen solution for injection was prepared by mixing lidocaine $(1 \mathrm{~mL})$ and gel-type atelocollagen $(1 \mathrm{~mL})$. During the procedure, upon detecting rotator cuff defect location by ultrasound examination, the solution was injected into the tendon defect by an in-plane technique. Total amount of injection was varied according to tear size present in each patient to prevent the volume effect, which can induce pain after injection. We stopped injection when the defect was full of atelocollagen materials based on ultrasound finding. Hence, the moment of injection cessation was determined by the practitioner. After the injection procedure, we recommended resting without rehabilitation treatment and prohibited anti-inflammatory medicine, such as nonsteroidal anti-inflammatory
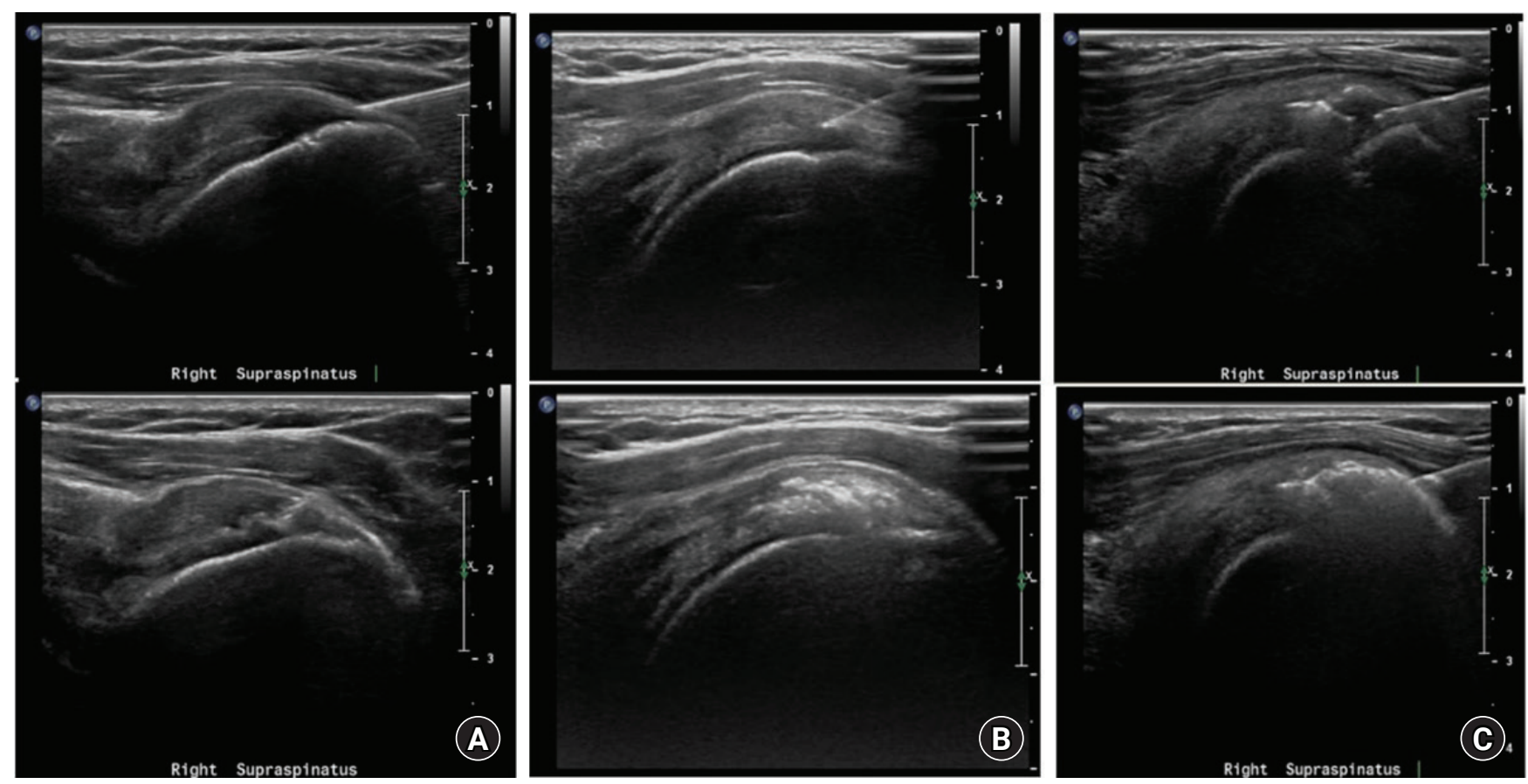

Fig. 1. Ultrasound-guided atelocollagen injection procedure on the (A) articular-side tear, (B) intra-tendinous tear, and (C) bursal-side tear. 
drugs (NSAIDs) or glucocorticoid, since they can relieve inflammation, which is the first phase of tendon healing [9].

We examined range of motion (ROM), visual analog scale (VAS) results for pain and function, and patient-reported outcome scores (American Shoulder and Elbow Surgeons [ASES], Simple Shoulder Test [SST], Korean Shoulder Score [KSS]) before and 2 months after injection. We performed ultrasound examination and assessed the status of tendon healing at a 2-month follow-up for seven patients $(46.6 \%, 7 / 15)$.

\section{Statistical Analysis}

The statistical analysis was conducted in IBM SPSS ver. 25.0 (IBM Corp., Armonk, NY, USA). For the nominal scale of demographics, the chi-square test was used. The Wilcoxon signedrank test was used to compare the clinical outcomes of pre- and post-injection. The level of significance was set at $\mathrm{P}<0.05$.

\section{RESULTS}

From May 2019 to September 2019, a total of 42 patients with partial rotator cuff tear underwent ultrasound-guided atelocollagen injection, and 15 patients were included under the criteria of patient selection. The 15 study patients consisted of five males and 10 females, with a mean age of 54.9 years (range, $40-72$ years). There were six patients with articular-side tear, three patients with intra-tendinous tear, and six patients with bursal-side tear. The mean follow-up interval after injection was 2.1 months (Table 1).

At the 2-month follow-up, ROM showed no significant difference compared to baseline. Clinical scores showed no significant change except in SST score $(\mathrm{P}=0.046)$ and function-visual analog scale (F-VAS; P = 0.009) (Table 2) [10]. After atelocollagen injection, a complication of pain was common. Eight of 15 (53.5\%) patients reported moderate $(n=2)$ to severe pain $(n=6)$. However, this injection-induced pain subsided approximately 2.5 days later according to the interview carried out at the 2-month follow-up. As there was no need to perform another procedure, such as steroid injection, NSAID medication was recommended to reduce residual pain.

Seven of 15 patients (46\%) underwent follow-up ultrasound to observe the healing status of the rotator cuff tendon. We found hyperechoic remnants likely to be atelocollagen in three of seven (42\%) patients (Fig. 2). In the four patients without remnants, there was no change in tear size.

\section{DISCUSSION}

This preliminary study showed no significant change in terms of ROM and clinical scores, except F-VAS and SST score $(\mathrm{P}=0.009$,

Table 1. Patient demographics

\begin{tabular}{lc}
\hline Variable & Value \\
\hline Age (yr) & $54.9 \pm 11.5$ \\
Follow-up period (mo) & $2.1 \pm 0.5$ \\
Sex & \\
$\quad$ Male & $5(33.3)$ \\
$\quad$ Female & $10(66.6)$ \\
Affected side & \\
$\quad$ Right & $13(86.7)$ \\
$\quad$ Left & $2(13.3)$ \\
Tear site & \\
$\quad$ Articular side & $6(40)$ \\
Intra-tendinous & $3(20)$ \\
$\quad$ Bursal side & $6(40)$ \\
\hline
\end{tabular}

Values are presented as mean \pm standard deviation or number (\%).

Table 2. Range of motion and clinical outcomes

\begin{tabular}{lcccc}
\hline Variable & Before injection & After injection $(2 \mathrm{mo})$ & Improved & P-value \\
\hline Forward elevation & $145.7 \pm 17.4$ & $154.3 \pm 19.1$ & 8.57 & 0.213 \\
Abduction & $140.7 \pm 24.6$ & $145.0 \pm 19.9$ & 4.28 & 0.224 \\
External rotation & $55.0 \pm 15.1$ & $61.4 \pm 16.6$ & 1 & 0.720 \\
Internal rotation (10-score) & $7.4 \pm 2.4$ & $8.4 \pm 2.5$ & -0.48 & 0.084 \\
P-VAS & $4.2 \pm 1.3$ & $3.7 \pm 1.1$ & 0.8 & 0.081 \\
F-VAS & $6.3 \pm 1.3$ & $7.1 \pm 1.2$ & 3.33 & 0.009 \\
ASES score & $57.0 \pm 10.7$ & $60.4 \pm 10.8$ & 2.5 & 0.125 \\
Constant & $56.4 \pm 12.3$ & $58.9 \pm 13.3$ & 3.9 & 0.224 \\
KSS & $64.6 \pm 12.2$ & $68.5 \pm 12.2$ & 0.29 & 0.169 \\
SST & $6.6 \pm 1.8$ & $6.9 \pm 2.4$ & & 0.046 \\
\hline
\end{tabular}

Internal rotation range of motion was converted to a 10-point scale [10].

P-VAS: pain-visual analog scale, F-VAS: function-visual analog scale, ASES: American Shoulder and Elbow Surgeons, KSS: Korean Shoulder Score, SST: Simple Shoulder Test. 
$\mathrm{P}=0.046)$, at 2 months of follow-up. More than half of the participants (53.5\%) complained of pain after injection, which subsided after 2.5 days. According to ultrasound follow-up, less than half of patients $(3 / 7,42 \%)$ showed hyperechoic material, which was presumed as atelocollagen remnants.

A few difficulties were encountered during the atelocollagen injection procedure. First, the optimal amount of atelocollagen injection has not been established. Since the defect size of the partial rotator cuff tear was different from person to person, patients with a small defect complained of severe pain upon injecting the entire dose $(2 \mathrm{~mL})$ of atelocollagen solution. Therefore, except for the first few patients, solution amount was based on tear size (Fig. 3). Second, it was difficult to differentiate partial tear from tendinosis by ultrasound or MRI [11]. It is known that tendinosis shows focal hypoechoic swelling, whereas tendon tears are more linear, hypoechoic, and sharper in outline [12]. However, in some patients, excess pressure was required during injection at the tear site. We believe that this increased pressure might be due to failure of differentiation between tear and tendinosis by ultrasound.

Previously, Martinello et al. [13] showed successful recellularization of human tendon using atelocollagen scaffold, and Suh et al. [8] presented a biomechanical and histological study with better healing of the rotator cuff tendon in a rabbit model. Although those studies were positive and offered encouraging results on the effect of atelocollagen, the experimental setup differs from that in the present study. We performed ultrasound-guided atelocollagen injection in an outpatient clinic without additional stem
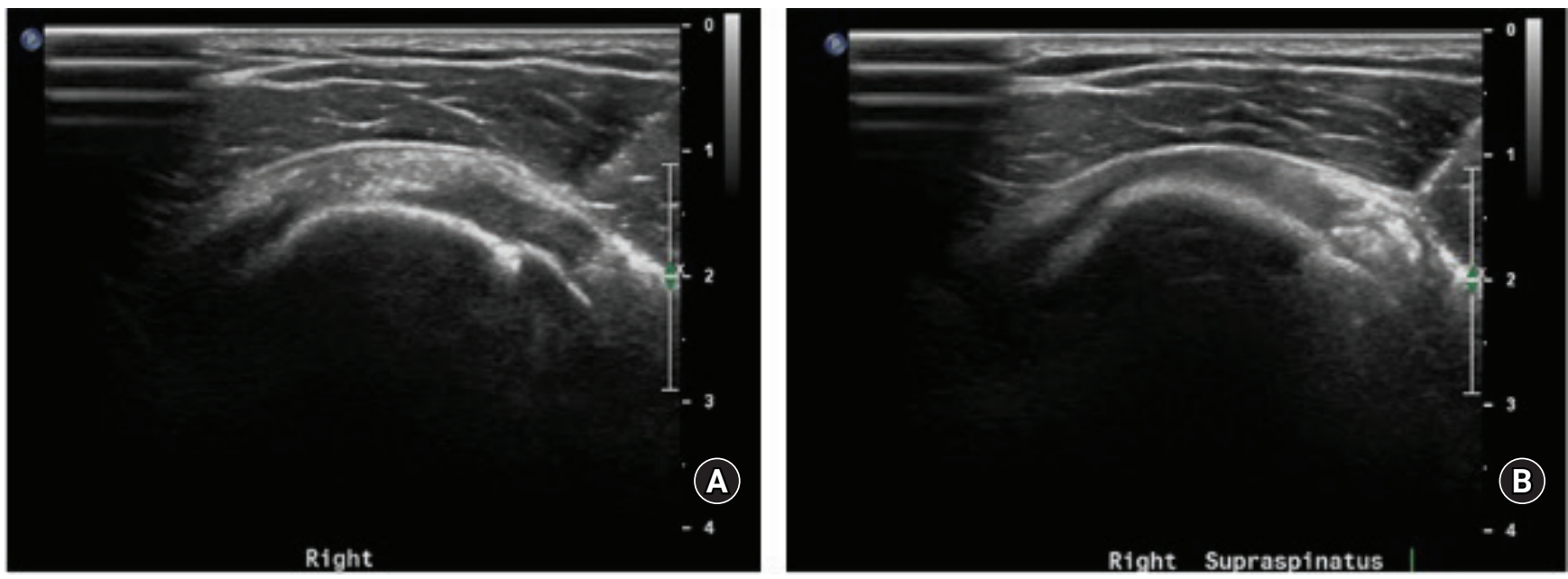

Fig. 2. (A) Ultrasound finding of bursal-side tear in the supraspinatus (transverse plane). (B) Ultrasound at 2 months after atelocollagen injection (transverse plane).
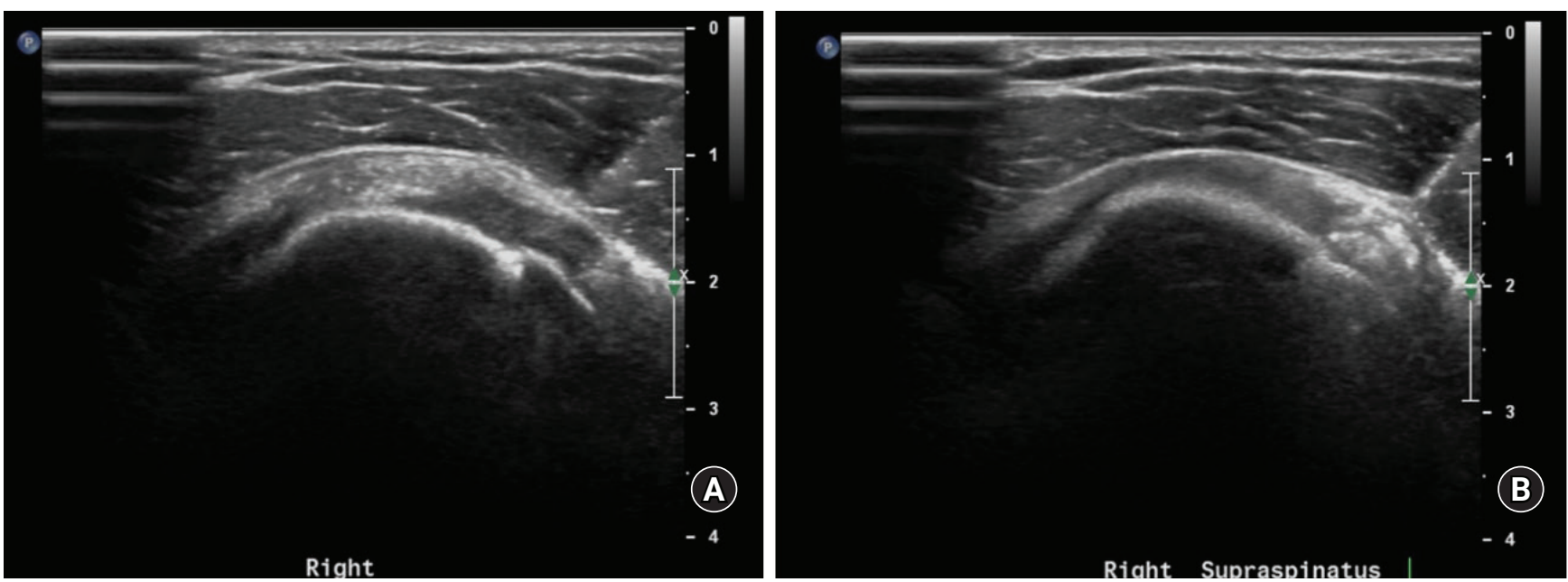

Fig. 3. Atelocollagen injection based on defect size. Before (A) and after (B) injection according to the size of the defect. 
cell or bone marrow stimulation. Martinello et al's method [13] used adipose-derived mesenchymal stem cells with collagen gel, and Suh et al.s method [8] used an atelocollagen patch for rotator cuff repair by transosseous equivalent technique.

It is not clear whether atelocollagen injection and bleeding from needle trauma are sufficient for tendon healing. One of the most important characteristics of ultrasound-guided injection is lack of bone marrow stimulation after the atelocollagen injection procedure. According to the natural healing process of tendon tears, the first inflammation stage begins with formation of hematoma, which could be caused by tendon injury [14]. Although a previous study [15] claimed that needle trauma can initiate the healing process with a small amount of bleeding, tendon to bone healing is more difficult than bone to bone healing [16]. Therefore, we believe the bleeding from needle trauma might be insufficient to heal the tendon.

One important complication after injection was post-injection pain. Eight of 15 patients (53\%) complained of pain. Among the eight patients, three suffered from pseudo-paresis due to severe pain after injection, although it recovered spontaneously after 2 to 3 days. We thought the pain might result from inflammation due to healing, like in the mechanism of prolotherapy [15]. However, we could not identify healed tendon upon ultrasound examination at the 2-month follow-up. Although we found hyperechoic deposits in three of seven (1, bursal side injection; 2 , intra-tendinous injection) patients, we interpreted hyperechoic deposits observed by ultrasound to be remnants of atelocollagen. We found no correlation between hyperechoic remnants and post-injection pain as none of the patients with hyper-echoic signals complained of pain. Regarding the tendon healing mechanism, we think a 2-month follow-up period may be insufficient for proper analysis. Considering the natural tendon healing process and features of atelocollagen as a scaffold for cell proliferation, we believe a healing period of at least 4 to 12 months is needed $[14,17,18]$.

Our study has several limitations. First, there could be a technical bias since atelocollagen injection was performed by three orthopedic fellows. Only one of them (SHC) is included as an author. For that reason, we excluded the first 10 consecutive patients. Second, since this is a preliminary study, the follow-up period was short (2 months) and number of patients was small. Based on our research, further study is needed to elucidate the effect of atelocollagen injection. Third, there could be a technical bias because three practitioners of shoulder orthopedics performed baseline and 2-month follow-up sonography. However, simply exclusion of 10 consecutive patients cannot assure that we have eliminated technical bias. Fourth, the patients in this study may have had tendinosis misdiagnosed as partial tear. We cannot rule out the possibility that post-injection pain is related to tendinosis.

Ultrasound-guided atelocollagen injection for partial rotator cuff tear showed no significant change in clinical outcomes except F-VAS and SST score. Tendon regeneration was not clear due to remnants of atelocollagen at 2-month follow-up ultrasound. We recommend warning patients about post-injection pain for 2 to 3 days before atelocollagen injection in an outpatient clinic.

\section{ORCID}

Sang Hoon Chae

Jae Yeon Won

Jae Chul Yoo https://orcid.org/0000-0002-7143-5074 https://orcid.org/0000-0003-4655-2540 https://orcid.org/0000-0001-8378-1583

\section{REFERENCES}

1. Yamanaka K, Fukuda H. Pathological studies of the supraspinatus tendon with reference to incomplete thickness tear. Katakansetsu 1987;11:98-102.

2. Lohr JF, Uhthoff HK. The pathogenesis of degenerative rotator cuff tears. Orthop Trans 1987;11:237.

3. Fukuda H. Partial-thickness rotator cuff tears: a modern view on Codman's classic. J Shoulder Elbow Surg 2000;9:163-8.

4. Yamanaka K, Matsumoto T. The joint side tear of the rotator cuff: a followup study by arthrography. Clin Orthop Relat Res 1994;304:68-73.

5. Sano A, Maeda M, Nagahara S, et al. Atelocollagen for protein and gene delivery. Adv Drug Deliv Rev 2003;55:1651-77.

6. Sakai D, Mochida J, Yamamoto Y, et al. Transplantation of mesenchymal stem cells embedded in Atelocollagen gel to the intervertebral disc: a potential therapeutic model for disc degeneration. Biomaterials 2003;24:3531-41.

7. Gomoll AH, Kamei G, Ochi M, Shetty AA, Zaslav K. Technical enhancements and update on chondrocyte implantation. Oper Tech Orthop 2014;24:35-47.

8. Suh DS, Lee JK, Yoo JC, et al. Atelocollagen enhances the healing of rotator cuff tendon in rabbit model. Am J Sports Med 2017;45:2019-27.

9. Kobayashi M, Itoi E, Minagawa H, et al. Expression of growth factors in the early phase of supraspinatus tendon healing in rabbits. J Shoulder Elbow Surg 2006;15:371-7.

10. Triplet JJ, Everding NG, Levy JC, Moor MA. Functional internal rotation after shoulder arthroplasty: a comparison of anatomic and reverse shoulder arthroplasty. J Shoulder Elbow Surg 
2015;24:867-74.

11. de Jesus JO, Parker L, Frangos AJ, Nazarian LN. Accuracy of MRI, MR arthrography, and ultrasound in the diagnosis of rotator cuff tears: a meta-analysis. AJR Am J Roentgenol 2009;192:1701-7.

12. Cholewinski JJ, Kusz DJ, Wojciechowski P, Cielinski LS, Zoladz MP. Ultrasound measurement of rotator cuff thickness and acromio-humeral distance in the diagnosis of subacromial impingement syndrome of the shoulder. Knee Surg Sports Traumatol Arthrosc 2008;16:408-14.

13. Martinello T, Bronzini I, Volpin A, et al. Successful recellularization of human tendon scaffolds using adipose-derived mesenchymal stem cells and collagen gel. J Tissue Eng Regen Med 2014;8:612-9.

14. Docheva D, Muller SA, Majewski M, Evans CH. Biologics for tendon repair. Adv Drug Deliv Rev 2015;84:222-39.
15. Kim SR, Stitik TP, Foye PM, Greenwald BD, Campagnolo DI. Critical review of prolotherapy for osteoarthritis, low back pain, and other musculoskeletal conditions: a physiatric perspective. Am J Phys Med Rehabil 2004;83:379-89.

16. Ouyang HW, Goh JC, Lee EH. Use of bone marrow stromal cells for tendon graft-to-bone healing: histological and immunohistochemical studies in a rabbit model. Am J Sports Med 2004;32:321-7.

17. Cole B, Lam P, Hackett L, Murrell GA. Ultrasound-guided injections for supraspinatus tendinopathy: corticosteroid versus glucose prolotherapy - a randomized controlled clinical trial. Shoulder Elbow 2018;10:170-8.

18. Lipman K, Wang C, Ting K, Soo C, Zheng Z. Tendinopathy: injury, repair, and current exploration. Drug Des Devel Ther 2018;12:591-603. 